\title{
Las historias de vida en el método de planificación pastoral ver-juzgar-actuar
}

\author{
Luigi PeLLEGRINO \\ Diócesis de Taranto (Italia) \\ luigi22272@gmail.com
}

\begin{abstract}
Resumen
Es bien conocido el aprecio que desde hace tiempo tiene la Iglesia por el método ver-juzgar-actuar, sin embargo, las reflexiones de la filosofía y la sociología hacen considerar hoy la necesidad de enriquecer el momento del ver, con unos elementos fenomenológicos y hermenéuticos que lo hagan más histórico y menos objetivista y de lograr además una mayor interacción entre los tres momentos. En este sentido, la investigación propone la inserción de las historias de vida de las personas y las comunidades como un camino nuevo de conocimiento de la realidad. En efecto, la realidad no sólo consiste en el hecho desnudo, sino también en el hecho tal y como lo percibe el sujeto; no sólo es suceso, sino también narración. Así, las historias de vida, y el consecuente método autobiográfico constituyen, según la investigación, un recurso vital en la planificación pastoral; no simplemente un apéndice secundario o un recurso simplemente aplicativo, sino, un momento intrínseco de la reflexión y de la experiencia teológica y pastoral.
\end{abstract}

Palabras clave: Planificación, método pastoral, ver-juzgar-actuar, historias de vida, autobiografía.

\section{Life stories in the pastoral planning method see-judge-act}

\begin{abstract}
It is well-known the log lasting appreciation of the church for the method see-judge-act, however the current philosophical and sociological considerations are considering the need for an enrichment of the moment of seeing with some phenomenological and hermeneutical elements which could make it more historical and less objective and to achieve, moreover, a major interaction among the three moments. In this sense, the research proposes also the introduction of the stories of people's lives and of the communities as a new way of awareness of reality. Indeed, reality is not only in the naked event, but also in the event as it is seen by the subject; not only the happened event, but also its narration. For this reason, the life stories and the autobiographical method are, according to the research a vital resource in the pastoral planning; not simply a secondary appendix or a applicative type, but as an intrinsic moment of the theological and pastoral reflection and experience.
\end{abstract}

Key words: Planning, pastoral method, see-judge-act, lifestories, autobiography.

Presbítero. Licenciado en teología pastoral por la Universidad Lateranense de Roma (2001). En 2014 se graduó en Filosofía con orientación en Psicopedagogía en la Universidad de Roma - Tor Vergata. Doctor en Teología por la Universidad Pontificia Bolivariana (Colombia).

Recibido: 15/Diciembre/2016 - Aceptado: 19/Enero/2017 


\section{Introducción}

Es innegable que ahora el mundo con sus procesos de desarrollo, con la innovación de la tecnología, con los movimientos de los pueblos no sólo de sur a norte, sino también de este a oeste, está cambiando rápida y profundamente.

La Iglesia, en este mundo que cambia, está llamada, obviamente, a decidir la manera como debe caminar en el mundo: no puede ser un caminar exclusivamente delante o detrás, y mucho menos ponerse de frente mostrando contraposición. Así, la única posibilidad que le queda, es caminar junto al mundo, sin confundirse ciertamente, pero mezclándose como la levadura en la masa sin miedo a perder su identidad. Por esta razón, el caminar de la Iglesia, para que no pierda la dirección y la orientación, tiene que ser concebido y planificado de una manera dinámica, involucrando el mayor número posible de actores en la planificación pastoral.

Es aceptado de manera unánime, por lo menos entre los expertos (teólogos, pastoralistas y agentes de pastoral), el valor del método participativo como herramienta fundamental para el cambio de rutas y la opción por caminos significativos. Ese método participativo ha valorado tres momentos fundamentales: ver, juzgar y actuar, que por supuesto también esta investigación valora. Sin embargo, los avances de la filosofía y la sociología nos hacen preguntarnos, si acaso no será necesario aplicar los nuevos descubrimientos de la fenomenología y la hermenéutica al método para hacerlo más histórico y menos positivista, menos ontológico y más fenoménico.

\section{La planeación participativa: origen y momentos}

Planificar significa elaborar y pensar propuestas que sepan responder a dos preguntas que la realidad nos plantea: ¿qué es lo que hay que hacer?, y luego, ¿cómo hay que hacerlo?, esto en una doble referencia: a lo pensado y a lo teorizado.

El origen de la planeación participativa debe buscarse en las prácticas populares, eclesiales y ciudadanas de los años 70, y en la reflexión de intelectuales comprometidos con ellas donde todos los interesados podían y tenían que aportar algo de ellos mismos, estimulando una dinámica nueva de diálogo.

A este propósito el método participativo es un método, o una dinámica que hace crecer toda una comunidad, puesto que todos se hacen responsables del proceso emprendido. Es una manera nueva de ser parte de la realidad que se quiere cambiar y transformar.

La ventaja de la planificación participativa radica en que se tiene una 
lectura más efectiva y verdadera de la misma realidad y que la toma de responsabilidad por parte de los actores directos e indirectos del proceso participativo responde mejor al significado del proceso mismo. Además hay que considerar que el trabajo en equipo, como instrumento esencial para ser realmente factor de cambio en su contexto es mucho más eficaz respecto al que trabaja a solas, donde los demás son sólo piezas o peones que realizan un plan pre-establecido. De esta manera la propuesta o la solución que se propone es seguramente la más apropiada porque responde a una lectura lo más amplia posible. Podemos afirmar, entonces, que la planeación participativa, ya sea en el ámbito pastoral o en el ámbito social, está orientada a una praxis y a una acción transformadora.

El P. Jesús Andrés Vela, en una publicación denominada "El proceso de planificación participativa" (Vela, 1986), presenta un modelo de planeación donde la expresión "participativa" puede aplicarse a diferentes metodologías, en la medida en que muchas de ellas insisten en la necesidad de que los diferentes pasos de planeación sean realizados con la participación de muchas personas y grupos.

Este método participativo, según Agenor Brighenti (1994), comporta tres grandes momentos. El primero es una explicitación del marco referencial, donde hay que tomar en cuenta dos reseñas de preferencia explícitas: la realidad histórica (en que está inserto) y la utopía (a la cual se quiere reorientar esta misma realidad). Este marco está especificado en el de la "realidad", donde las personas involucradas, desde sus prácticas, expliciten y tematicen la realidad en la cual están insertas; en lo "doctrinal", donde de manera dialéctica hay una mutua confrontación entre revelación y situación, y se proyecta el futuro deseable para ser seguido por la acción pastoral; $y$, por último, en el "diagnóstico pastoral" como resultado de la confrontación entre el marco de la realidad y el marco doctrinal.

El segundo momento es el de la elaboración del marco operacional que crea el camino descendente, de regreso a la acción. Se despliega en dos momentos: el "pronóstico pastoral", que establece el objetivo general del plan global y los objetivos específicos, diseñándose el curso de acción, con sus criterios de acción; y la "programación pastoral" que define campos de acción y acciones y metas concretas.

El tercer momento, el de la ejecución del plan, elaboración que evita que lo planeado sea un asunto sólo de papel. Es una concreción que se da a través de la organización institucional donde se piensan los mecanismos de coordinación, a través del seguimiento o control para evitar errores, y a través de la evaluación de lo realizado.

A tal propósito, el método ver-juzgar-actuar, refleja teórica y prácticamente este modelo de planificación participativa, donde todos los sujetos 
involucrados en los distintos niveles son partes activas del proceso de planificación.

De hecho, el primer momento del método ver-juzgar-actuar corresponde a la necesidad de saber en dónde estamos, encuadrando el marco de la realidad que orientará todo el sistema de planificación. El primer momento, el "ver", se queda en la realidad, investigándola sin violencia, más bien acogiéndola para comprenderla y reorientarla hacia el futuro. Por eso es importante el elemento, o mejor dicho, la dinámica participativa, porque el futuro es de todos los involucrados en el mismo proyecto. Aquí, método, proceso, planeación encuentran un punto en común que resulta ser la realidad vivida (participada) y animada.

\section{Método participativo y magisterio eclesiástico}

En el ámbito eclesial ese elemento/dinámica es una manera de dar forma histórica a aquél modelo eclesial de comunión, propia del Concilio Vaticano II, que ve en la participación del pueblo de Dios en la construcción del Reino, el elemento de identidad propio de la Iglesia. El modelo de planificación participativo que se hace histórico en el método ver-juzgar-actuar, responde de manera explícita a la exigencia que cada hombre, con su propia historia personal, es sujeto activo y responsable, junto con los demás, en el construir un mundo diferente.

El trinomio ver-juzgar-actuar significa, en síntesis, un método de planificación pastoral, que nació como método de la acción en el seno de la JOC belga, usado también en los documentos de la Iglesia, y más precisamente por Juan XXIII en Mater et Magistra, en 1961. En seguida con la Gaudium et Spes (promulgada por el papa Pablo VI el 8 de diciembre de 1965, último día del Concilio) se transforma en método teológico, y más tarde se convierte en un principio arquitectónico de los documentos conclusivos de las Conferencias generales de la Iglesia latinoamericana.

El método tuvo un gran éxito por ser inductivo, porque partía de la situación, alejándose de los métodos tradicionales deductivos, que de ideas generales y universales deducían lo que se debía hacer.

El "ver" propone un hecho de vida con el fin de descubrir actitudes y modos de pensar y valores y comportamientos. Se buscan las causas y se analizan las consecuencias que pueden tener en las personas, en las comunidades, en las organizaciones sociales. Se invitaba a los jóvenes obreros a revisar su vida en el trabajo, la familia y la sociedad.

El "juzgar" es el momento central de la revisión de vida. Se propone tomar posición frente al hecho analizado, explicitar el sentido que descubre la fe, la experiencia de Dios que conlleva y la llamada a la conversión que surgen de él. Se trata de un discernimiento. 
El "actuar" se propone determinar aquellas actitudes que las personas deben cambiar en sus vidas, los criterios de juicio que deben ser transformados, los hábitos que son cuestionados por la Palabra de Dios y las acciones que se van a desarrollar (Castillo, 2004: 34).

El "ver", como marco de la realidad, así como los otros dos momentos (juzgar y actuar), necesita de la participación de todos para que la realidad sea realmente reflejada en forma fiel y profunda, pues a partir del "ver" se irá implementando todo el resto de la planificación en su juzgar y actuar.

\section{Límites del método participativo}

Hay que decir que no existe un método absoluto y único en la planificación pastoral. La decisión de utilizar un método más que otro depende de lo que se quiere obtener al final del proceso. Por lo que afecta al método del ver-juzgar-actuar creemos que, a veces, si no siempre, los límites se refieren no tanto a su estructura teórico-práctica, sino al uso que se hace en la práctica y además las modalidades a través de las cuales se declina en el nivel empírico operativo el momento del "ver" que - para nosotrosno viene asumido y actuado en toda su realidad teológico-práctica.

Pero, por lo que se refiere a la literatura vigente, no faltan debilidades y límites. Entre éstos nos han parecido más afines a nuestra investigación aquellos puntos que subrayan, por un lado, la relatividad del método mismo y, por el otro, la necesidad que sea ampliado y reforzado por parte de otros elementos.

Entre los riesgos mayores, nos parece poder colocar ante todo lo que normalmente llamamos yuxtaposición de las partes. El método tiene una coherencia interna, en la que cada fase viene después de la anterior. La realidad analizada en el "ver" es interpretada en el "juzgar", y del diagnóstico que se deriva de la comparación del "ver" con el "juzgar" emerge el "actuar". Resulta una yuxtaposición, un "poner algo junto a otra cosa" sin guardar ninguna relación interna, donde una cosa no guarda relación con la otra. Hay secuencia sin consecuencia. Cada parte sigue a la otra, pero no hay lógica interna. Hay un agregado de partes, pero no un sistema estructurado y coherente (Castillo, 2004).

Sobre todo, en el método ver-juzgar-actuar se produce con frecuencia una neta separación entre el momento analítico y el propio de la planificación. El análisis y la evaluación de la situación, de hecho, no influyen en la determinación de los objetivos y de las decisiones que se encuentran en el ámbito de la fe en forma pre-escrita e independiente del contexto (mientras, en realidad, la fe determina los principios y los criterios).

Raramente se determinan las prioridades pastorales por medio de una evaluación de la situación, bien conectada a la misma situación y contexto: 
la pastoral está llamada a decidir lo que es más importante y urgente no en sí misma, sino más bien en relación con las exigencias reales de aquella comunidad o de aquella persona: poniendo entonces en relación de reciprocidad los principios y los valores absolutos con la situación del campo (Lanza, 2010).

Otro elemento de debilidad lo podemos enfocar acerca del "juzgar". Muchas veces el "juzgar" no guarda ninguna relación con la realidad analizada en el "ver". Se presenta como una bella síntesis teológica sobre un tema, como una declaración de principios aséptica, neutra y fría. El resultado es que el "juzgar" deja de ser lo que es; en otras palabras, el "juzgar" no juzga, no dice nada sobre la situación, no la califica, no la interpreta. Se habla "de un extrincesismo teológico porque el juzgar se convierte en un conjunto de enunciados teológicos totalmente extrínsecos a la realidad vista y analizada" (Castillo, 2004: 44).

Algunas veces el "juzgar", que es eminentemente teológico-pastoral, no considera la estructura humana, cultura, ética y filosófica. Por eso el juicio teológico es extrínseco, no considera el principio de encarnación: lo que no es asumido, no es redimido. Lo teológico es externo, es un paraguas colocado sobre una situación dada; no asume la mediación de lo humano, más bien se sobrepone a ella. El "juzgar", como hemos dicho anteriormente, debe ser una interpretación teológico-pastoral de la realidad. Debe hacer una lectura a la luz de la Palabra de Dios.

Otra debilidad es la que llamamos apriorismo de la práctica, consecuencia de la yuxtaposición de las partes. El "actuar" debe indicar lo que hay que hacer para que la realidad descrita en el "ver", el perfil real, se parezca cada vez más al sueño expresado en el "juzgar”, al perfil ideal. De aquí la imperiosa necesidad de que el "actuar" emerja del diagnóstico (Castillo, 2004).

Si esto no sucede, nos encontramos, entonces, con el apriorismo de la práctica. El actuar no se induce de la valoración e interpretación de la situación, sino que se propone independientemente, pre-fijados que se aplican en cualquier situación y tiempos diversos.

El inmediatismo pastoral conlleva frecuentemente a un vacío de pensamiento, a un no dar importancia al "juzgar" como momento teológicopastoral y de aquí se pasa a una lectura de la realidad que no es real, porque se carece de los instrumentos adecuados para el análisis. La supremacía de la práctica lleva a la aridez del "juzgar" y a la miopía del "ver": "apriorismo de la práctica y tiranía del inmediatismo pastoral son dos aplicaciones no correctas del método y dos trampas que amenazan con reducir la eficiencia de la promoción humana y cristiana, y la fuerza de la praxis evangelizadora y libertadora" (Castillo, 2004: 47).

El método ver-juzgar-actuar separa claramente el "ver" (a menudo 
propio de la sociología) del "juzgar” (dejado a la teología entendida como dogmática); pero no se da ni se puede dar un "ver" que se ponga sobre un nivel solamente descriptivo, sin involucrar, en cambio, desde su comienzo, una precisa perspectiva de lectura (pre comprensión/interés) y una, por lo menos inicial (aunque no consiente) activación de criterios de interpretación.

Esta aporía es tanto más peligrosa cuando encarga a la sociología la tarea de tomar foto y decir la verdad: no existe sociología neutral y unívoca. La pretensión de neutralidad lleva, de hecho, a una posición inconscientemente ideológica. Se propone como dado lo que en realidad es el resultado de una lectura del mismo.

La respuesta incluye, de alguna manera, la pregunta que la orienta. Se habla de ideología en cuanto se da valor de dado al ver, como si no fuera una visión e interpretación del dado que se debe examinar críticamente. Parafraseando una expresión de Gabriel García Márquez (2002), la realidad no es la que se ve, sino la que se lee para poderla contar ${ }^{1}$.

\section{Consideraciones útiles para superar los límites}

Para superar las diferentes dificultades o anomalías internas propias del método, garantizando al mismo tiempo su eficacia, creemos necesario recuperar una lectura y una visión de la realidad que, además de ser dada y darse, pueda orientar caminos de planificación pastoral. De esta manera, convertir la misma visión de la realidad en relato viviente, capaz de superar las dificultades e incertidumbres interpretativas porque es alimentado por una verdad que sale directamente de su capacidad de contarse y de nuestra capacidad de escuchar, de esta manera podemos llegar hasta el final del proceso metodológico propuesto por el "ver-juzgar-actuar".

Esto permitirá acompañar el proceso, motivarlo, apoyarlo y de esa manera dejar que decline el relato de una historia nueva: la transformación de la realidad, fin último de toda planificación.

El texto de Éxodo 3,7 nos ayuda a enriquecer el momento del conocimiento de la realidad preliminar al juzgar y actuar donde Dios mismo hace del ver, junto al escuchar, una actitud primaria para vivir su proyecto de salvación para su pueblo herido y sufriente. En el texto se ve un Dios que libera, con una metodología que la misma Iglesia latinoamericana, ha asumido en los últimos 60 años: "ver" (Yo he visto la opresión de mi pueblo), junto a la "escucha" (he oído los gritos de dolor), "juzgar" (conozco muy bien sus sufrimientos) para tomar decisiones de valor, y luego sigue

1 La oración original está presente en el libro del 2002 "Vivirpara contar": "La vida no es lo que uno vivió, sino la que uno recuerda y cómo la recuerda para contarla".

VERITAS, No 36 (Abril 2017) 
el "actuar" como proceso de planificación de liberación (Por eso he bajado a librarlo).

La lógica del Éxodo, es la lógica de Dios, que resulta ser legítimamente evocativa para que sea nuestra lógica de Iglesia. El relato del Éxodo no está terminado, no está cerrado ni concluido; todavía muchos gritos esperan la escucha a través de procesos de liberación que Dios nos obliga a actuar en nombre de una alianza viva, y por eso sigue el texto con "ponte en camino".

"He visto (...), he oído (...), conozco (...), he bajado a librarlo" (Ex $3,7)$ es un camino obligado que desde el Éxodo continúa señalando procesos únicos de liberación, caminos de evangelización.

El conocimiento (ver: como primer momento de la trilogía) que Dios se procura de la realidad está realizada por el "ver" y el "escuchar", donde el escuchar es la actitud de Dios de dejarse contar y narrar por medio de lágrimas las historias cotidianas de su pueblo.

Por eso es importante recordar que el uso del "ver", como primer paso de la planificación pastoral, no es solamente una técnica para recaudar informaciones, sino también el momento kayrológico ("he oído", "he conocido") en que la realidad de las personas, la historia socio-eclesial de las comunidades sea parte integrante de un proyecto y proceso de liberación, como expresión de historia de salvación. Concretamente se trata de recibir el testimonio de la planificación que Dios nos entrega con confianza y responsabilidad. A eso tiene que orientarse el mismo método: fiel al deseo de Dios de liberar y fiel en el responder a las expectativas del hombre, de las comunidades humanas y cristianas.

Varias veces en el uso del método ha faltado el momento sincrónico, el pasar de ver a juzgar y actuar son momentos que necesitan un tiempo, el mismo tiempo que el mundo y su realidad utilizan para caminar y transformarse con el riesgo de que nuestro análisis, nuestro ver, sea solamente nuestro, y ya no respondiente y partícipe de la realidad misma.

Aunque el método del ver-juzgar-actuar ha ayudado a las comunidades a encontrar caminos de liberación haciendo, entonces, parte del trabajo pastoral, estando presente en la vida de muchas Iglesias particulares, parroquias y de modo más constante en las Comunidades Eclesiales de Base y Pastorales Sociales, se necesita recuperar una dimensión personal dentro de la comunidad que acompañe todas las etapas del proceso de la planificación pastoral.

Es por eso que el momento del "ver", no puede ser solamente sustentado y caracterizado por instrumentos y técnicas sociológico-cuantitativas o simplemente confiando en unos conceptos de historia mecanicista y de una idea de la realidad causa-efecto (recordando que el método apunta 
sobre todo a la acción). Debemos considerar que hay elementos, dinámicas, vidas que no son previsibles, como por ejemplo la historia personal de cada sujeto, y la de varias comunidades, que son parte activa del proceso de transformación y de cambio con toda su historia personal y vivencial, que no siempre el método toma en cuenta.

No se dan hechos desnudos, sino siempre, cognoscitivamente, interpretados. También las ciencias sociales presentan resultados que son en realidad construcciones de segundo grado, comprensibles solamente en relación a las modalidades que la investigación ha llevado a la práctica. El "sujeto está implicado con su yo, con su cuerpo, con sus relaciones, con sus experiencias" (Klein, 1999: 251). Por lo tanto, podemos añadir que la lectura de la realidad no se detiene fragmentariamente en los hechos, sino en la búsqueda de una lógica interior que determinan los hechos, lógica que se manifiesta en ellos, y por ellos están modificados.

Se necesita una dinámica interna al mismo método que pase de las estructuras a las personas y a las comunidades, capaz de escuchar, sanar, curar, transformar no solamente al término del proceso teológico, sino más bien durante el proceso mismo; un proceso, entonces, que sea más narrativo, donde las personas puedan contar y relatar, como instrumento de recuperación del propio tejido existencial para un futuro de esperanza y de vida.

Esto lo aprendimos también a través del camino del desarrollo de la hermenéutica contemporánea, con la oportunidad de ponerse en contacto con autenticidad y sin atajos con las historias pasadas, que han de ser redimidas y añadidas al presente en el que se narran, se escuchan, se interpretan.

Los filósofos se han enfrentado en esta época contemporánea con la relación sujeto-objeto (la mirada y la cosa). En este debate, Husserl tiene como objetivo una revisión completa de la tradición filosófico-científica europea; revisión necesaria por la crisis espiritual en la que la Europa de final de siglo XIX parece haber caído. Esa crisis tiene como causas principales el objetivismo científico y el materialismo positivista. Con la llegada de la ciencia moderna, de hecho, la filosofía ha perdido progresivamente el rol de reina de las ciencias, es decir, de fin último y fundamento del saber.

Por eso las mismas ciencias, justo mientras aparecen en su máxima lozanía, en su máximo esplendor, van hundiéndose en una crisis moral: crisis de sentido y de perspectiva, crisis de valores y de objetivos. La humanidad científica europea, en su intento/esfuerzo de racionalizar el mundo y la vida, ha perdido justo el objetivo último de sus obras y va transformándose en esclava de sus mismos inventos tecnológicos (Sini, 
1986), es así que se redescubre el retorno a la conciencia, en su intencionalidad, que quiere conectarse al objeto. El conocimiento, entonces, se realiza en la interpretación de lo vivido, "se trata de ver, de dejarse impregnar por el mundo, suspendiendo el juicio, dando voz de nuevo al objeto, redescubriendo el sentido y el orden de las cosas que la modificación continua de los sistemas de referencia y de apoyo han hecho inciertos y problemáticos” (Bodei, 2001: 112).

En la perspectiva del "ver", también Gadamer (2000), considerado el fundador de la hermenéutica contemporánea, y que se caracteriza por la idea de "círculo hermenéutico", es decir, la circularidad de los procesos interpretativos ${ }^{2}$, nos ayuda a recuperar la narración de la historia que se quiere conocer, y a entender que ésta llega hasta nosotros del pasado, pasando por nuestra conciencia, sin que algún elemento se pierda. Nosotros somos ser, somos un proyecto tirado (según la idea de la cura de Heidegger $^{3}$ ), y cuando conocemos, inevitablemente interpretamos continuamente conforme a lo que fuimos, a lo que somos y a lo que querríamos ser.

El sociólogo y filósofo austríaco Alfred Schütz (1974) articula y especifica el mundo indistinto y unitario de la vida de Husserl. Él asume el aspecto de "provincias finitas de significado", dotadas todas ellas de autonomía. Cada una es un universo simbólico, virtualmente autosuficiente, en el que se permanece hasta que un trauma lo induce a superar los límites. Es el trauma de la crisis, de la pérdida de confianza, de la seguridad adquirida (Muzzetto, 2011), un impacto con una realidad vista y escuchada, expuesta al ojo y al oído del observador. La alternativa ya no es neta como entre pluralidad de los mundos y mundo en singular, entre vidas paralelas y vida única, entre identidad absoluta y uno, ninguno y cien mil. Al no

2 Generalmente en la literatura filosófica, con "círculo hermenéutico" después de Gadamer, se entiende el proceso que, dado un texto para ser interpretado, cabe señalar que el enfoque del estudioso sólo puede ser caracterizado como una pre-comprensión inevitable del texto dada por el contexto histórico y cultural en el que vive, por lo que el conocimiento es un intercambio constante entre las nociones por aprender y conceptos ya aprendidos, entre aprendizaje y actitud interpretativa. Así el conocimiento está necesariamente situado dentro de un determinando horizonte histórico y psicológico, el resultado de una estratificación circular de nociones.

3 La existencia, para Heidegger, es ser en el mundo, por lo tanto, ser entre los demás. La cura es la expresión de la relación entre el hombre y los demás y puede ser auténtica o inauténtica. La cura inauténtica quita a los demás sus curas dándoles directamente lo que necesitan; por lo tanto, se dirige a los objetos y no a los hombres (por ejemplo, llevar el pescado a alguien que no sabe cómo pescar) y es expresión de "estar juntos". La cura auténtica, en cambio, ayuda a los otros a asumir sus propias curas y luego a ser libre para realizar su ser (por ejemplo, enseñar a pescar a alguien que no sabe cómo pescar); es una expresión de "coexistir" (Leyte, 2006). 
haber una realidad única a respetar, reflejar y trascender, el único movimiento posible resulta ser el paso horizontal de un mundo vital a otro.

El conocimiento, entonces (el "ver"), es entendido como posibilidad dada, en las migraciones del sentido.

El "ver", más que una actitud y/u operación humana, es una condición del hombre, que se expresa en la posibilidad de hacer propio el sí del ser, es decir, el ser que se encarna en la historia y en la realidad concreta de los acontecimientos.

La comprensión del concepto de fenomenología pasa por Heidegger (1968) a través de una clarificación etimológica del término mismo. La expresión fenomenológica puede ser formulada como sigue en griego: legeintafainomena, donde, legein significa apofainestai. Fenomenología significa, entonces, apofainestaitafainomena: dejar ver por sí mismo lo que se manifiesta, así como se manifiesta por sí mismo. Dejar es dar una posibilidad, para que el ser se exprese a su manera, así como es, condición necesaria para que el sentido se comunique, pase, trasmigre, hasta el cognoscente. Aceptar la objetividad de los fenómenos antes de interpretarlos.

El mundo, a su vez, no siendo una cosa, sino el horizonte del trascender humano, es aquel ámbito o aquella gama de posibilidades hacia las que el hombre tiende. Distinto de las cosas porque no es una realidad simplemente presente, más bien, un relacionarse a las posibilidades, el ser humano, y solo él, existe en el sentido etimológico de la palabra, es decir estar fuera, sobrepasa la realidad simplemente presente, en la dirección de las posibilidades.

El problema ontológico puede ahora ser solucionado: lo que, en sentido sobresaliente, queda escondido, o se manifiesta sólo en manera contrahecha, no es éste o aquel ente, sino más bien el ser. La fenomenología es la manera de alcanzar y determinar demostrativamente lo que tiene que constituir el tema de la ontología. La ontología no es posible si no solamente como fenomenología (Heidegger, 1927).

Según Heidegger, la mirada del ser-para-sí cotidiano sobre el mundo es caracterizado por la búsqueda de la satisfacción. El ente que se encuentra, manifiesta, en este modo, la propia utilizabilidad (Heidegger, 1927). En Heidegger, el conocimiento de la cosa no se presenta ya como visión o acierto de la visión; "es más bien el hombre quien es mirado por el ente, es decir, por quien se abre al estar presente contenido con él. Mirado por el ente, sustentado por él, involucrado en sus contrastes y marcado por su discrepancia: ésta es la esencia de hombre en el periodo de la grandeza griega" (Heidegger, 1968: 89-90). El conocimiento del "ver" se realiza para Heidegger cuando el contar del ser se hace posible en su desvelamiento.

Sartre introduce en el ámbito de la conciencia y de la visión un elemento conflictivo. Sólo otra conciencia, la mirada de otro individuo, puede 
reificar la conciencia, puede solicitar su flujo. En Sartre al mirar el mundo, yo me abandono en él, me dejo absorber por las cosas como la tinta por el papel secante. Pero he aquí que, de repente, la mirada del otro me hurta de mi mundo: yo puedo captar el verde de la hierba que veo, pero no veo el verde como se le aparece a otro. Algo se me escapa, algo inquietante que limita mi libertad, "como si el mundo tuviera un sumidero, en el centro de su ser, y que se escurriese continuamente en este agujero" (Sartre, 1960: 325). El otro es siempre para Sartre un antagonista, símbolo de peligro, el infierno, aquél que, objetivándome a mí mismo, me remite a mí mismo.

Podríamos decir, como uno de los protagonistas de la novela La prórroga (Heidegger, 1927), que la mirada del otro, además de perturbadora, es la garantía de mi existencia, el testimonio de que no soy una nulidad, de que cuento algo. El otro y su narración nos revelan buena parte de la realidad.

Con Habermas, nuestro camino del conocimiento de la verdad, de la realidad, se hace acto. Con la teoría de la acción comunicativa, Habermas trata la fundamentación de las ciencias sociales, no sólo como evolución de la filosofía del lenguaje, sino que por su conexión con la Teoría crítica de la sociedad de la Escuela de Frankfurt. La verdad, según Habermas, no es una copia de la realidad a la cual se refieren los argumentos de los participantes en el discurso, sino que es un resultado consensual sobre el cual no actúa ninguna influencia que lo distorsione. Ese consenso se logra cuando se dan cuatro condiciones de validez aceptadas por todos los participantes: a) que el enunciado que hace un hablante sea comprensible; b) que el hablante sea fiable; c) que la acción pretendida sea correcta por referencia a un contexto normativo vigente; y d) que la intención manifiesta del hablante sea, en efecto, la que él expresa (Arrieta, 2009).

Cada uno de nuestros autores ha aportado en términos de reflexión para el desarrollo del ser en relación con su presentación, reconocimiento y por esto en orden a su narración. Hacer ver cómo en la interpretación del ser, y por lo tanto de la realidad, con todas las cuestiones planteadas por los autores mencionados, siempre hay, además de "ver", incluso el aspecto del revelarse gracias a la narración de las cosas y, en consecuencia, a su escucha. El ser con su realidad, se pone de manifiesto no sólo por lo que hace ver de sí, sino también por lo que nos cuenta de su existencia, de su vida, que se materializa en la historia y en la historia individual de cada persona.

Gracias al "círculo hermenéutico" el tiempo no es un obstáculo, pero se convierte en el lugar y el espacio de encuentro entre el evento y su relato, lo que lo lleva más cerca de nosotros. Este es un cuento vivo, en cuanto contando o escuchando interpretamos, haciendo nuestra la historia o las historias que se nos entregan. En la óptica del ver nos equipamos de un 
esquema seguro para leer cuidadosamente y fielmente la realidad que queremos conocer para ejercer un juicio y una acción cada vez más cerca de la realidad.

No se dan hechos desnudos, más bien siempre, cognoscitivamente, interpretados. El "sujeto está implicado con su yo, con su cuerpo, con sus relaciones, con sus experiencias" (Klein, 1999: 251). Por lo tanto, podemos añadir que la lectura de la realidad no se para fragmentariamente en los hechos, sino en la búsqueda de una lógica interior que determinan los hechos, lógica que se manifiesta en ellos, y por ellos están modificados.

\section{Las historias de vida: una propuesta para enriquecer el método participativo}

"Ver" pertenece a las dinámicas de la vida de cada hombre y, en la óptica de la escucha, a la manera de "intervenir" de Dios en la/s historia/s de salvación/es de la gente. Pero el "ver" de la trilogía ver-juzgar-actuar, en la planificación pastoral, exige un aporte peculiar que pueda favorecer efectivamente la realización operativa, no tanto en el orden de la mera aplicabilidad cuanto más con relación a la visión/misión, que tienen, de por sí, en la planificación pastoral. No se trata de ver lo que no hay, de repente justificando su presencia, y tampoco de transferir en la realidad analizada lo que se quiere ver. La realidad que hay que ver está ya en la misma realidad que es grávida, no solamente de lo vivido, tampoco sólo del presente, sino mucho más de futuro y de esperanza. Conocer la realidad significa saber "ver" y "escuchar" la manera como la realidad se muestra a nosotros para que la conozcamos.

Se necesita una dinámica interna al mismo método que pase de las estructuras a las personas y a las comunidades, capaz de escuchar, sanar, curar, transformar no solamente al término del proceso teológico, sino más bien durante el proceso mismo; un proceso, entonces, que sea más narrativo, donde las personas puedan contar y relatar, como instrumento de recuperación del propio tejido existencial para un futuro de esperanza y de vida.

Ya en micropedagogía encontramos unas pistas de reflexión que podemos asumir como elementos propios para que las historias de vida sean útiles a la dinámica del ver, como parte de la trilogía (ver-juzgar-actuar) en la planificación pastoral.

Responsabilidad de la micropedagogía es ocuparse de los particulares, tejidos en un contexto, que pertenecen a una trama más compleja. El particular nos lleva de nuevo a la totalidad-complejidad, y el fragmento, se convierte en el objeto de la investigación, ya que el particular no se refiere 
más que a sí mismo. De hecho, la micro-pedagogía no quiere decir pequeña pedagogía, sino más bien: pedagogía de lo que es directamente observable, que es divisible y que revela las conexiones con otras fracciones. Es micro-pedagogía, "un hábito de quedarse en lo insignificante aparente o en lo evidente, que no se manifiesta si no ampliándolo" (Demetrio, 1996: 85).

Su objetivo es, pues, para resumir:

-La búsqueda de lo invisible o lo que está más allá del alcance de las posibilidades de la vista común; -ampliar el fragmento de entrar en su mundo;

-descubrir redes y conexiones absolutamente imperceptibles a simple vista entre las partes de lo particular (la trama);

-entrar en contacto con lo observado y con él hacer más evidente el deseo o la necesidad de comprender y cambiar la situación.

Todo esto nos motiva y nos empuja a incluir las historias de vida como elemento propio e imprescindible para un conocimiento profundo de la realidad.

La característica adicional de la investigación cualitativa es su valor transformador inevitable. No sólo porque se hace investigación cualitativa para averiguar lo que es posible cambiar, porque la inclusión del investigador cambia de hecho la situación, agregando nuevos equilibrios o desequilibrios. El investigador, a pesar de la incertidumbre y la imprevisibilidad que acompaña a estos tipos de rutas, es llamado por un método riguroso a detectar los criterios ordenadores del material que irá recolectando y sistematizando, y los métodos de detección que deberán obedecer a un principio de coherencia interna.

La narración con el método de las historias de vida, no es solamente un apoyo al momento del "ver" de la trilogía ver-juzgar-actuar, más bien es un apoyo a todo el proceso de planificación pastoral: en el momento del discernimiento y en su práctica comunicativa, enfocando el papel protagónico de los sujetos involucrados, y como momento de vínculo en los distintos momentos de planificación.

La comunidad cristiana para obedecer a su misión, a razón de su "praxis comunicativa" (Mette, 1978), debe abrir sus ojos, agudizar su intelecto y comprender las realidades que esperan las personas, los grupos, en sus diversas categorías, en fin, toda la sociedad organizada. Sólo la escucha paciente y el estudio competente iluminan, ayudan a distinguir, colocando los acentos y relieves sobre los puntos medulares de las distintas situaciones, acogiendo las urgencias y haciendo descubrir los mecanismos escondidos de los fenómenos. Para esto sirve la planeación o planificación que, 
hoy con diversas metodologías se presenta: la Normativa, la Estratégica, la Perspectiva y la Participativa, aplicadas al ámbito de la acción pastoral, cada una de ellas es portadora de una eclesiología y mística que hacen inteligible la compleja realidad humana, poniendo a disposición interpretaciones que indican la relevancia, los valores y los límites (Brighenti, 2003). Pero sobre todo ayudan a la comunidad en el proceso de planificación a dar la palabra, a interactuar, viviendo en comunión entre los sujetos de la comunidad cristiana. Ahora bien, en este sentido planificar es obra de todo el pueblo de Dios, como quiere el Magisterio pastoral del Vaticano II y las sucesivas elaboraciones doctrinales.

Las historias de vida, no son sólo aquéllas del hombre de hoy, como sujeto que narra su vida, también lo es aquélla de Dios que continúa mostrándose en el aquí y ahora de nuestra realidad. El "ver", en la perspectiva del "juzgar", el discernir ahora, es hacer que la mirada y el oír del "ver", se confronten, se entrecrucen, se iluminen y, sobre todo, nos abran y despierten a los creyentes a la manifestación de la historia de Dios que se queda en las actuales comunidades cristianas; por eso es el Dios de la historia que va actuando en el aquí y ahora de nuestro pueblo. También Dios es una autobiografía que se manifiesta a través de los acontecimientos humanos y, no por eso, es menos verdad la realidad humana; Dios planifica la realidad humana. La autobiografía ayuda al discernimiento y al juzgar en cuanto utiliza el mismo lenguaje, aquel del contar o narrar, esto hace encontrar la realidad del hombre y la realidad de Dios, de manera que la una ilumina la otra y viceversa, aunque con tareas diversas.

La autobiografía de Dios pone a disposición los criterios y las condiciones sobre las cuales acoger y analizar las autobiografías humanas como un ligamen necesario, porque Dios mismo tiene significado en los procesos de planificación y transformación de la realidad. Podríamos decir que es la historia de los hombres que se orienta al contar o narrar la historia de Dios; ésta da verdadero sentido a la historia humana ya que la gloria de Dios es el hombre viviente.

Aquí, sin caer en una crítica relativista o de debilidad de Dios, no se está hablando de su esencia, sino de su auto-narrarse o darse a conocer, que, en vez de cortar, agrega términos de cercanía y de significados necesarios para su comprensión en términos de su acogida, aceptación, no racionales, sino existenciales.

En el asumir el aspecto narrativo-biográfico, otra implicación sobre el "ver", ligado al actuar eclesial (sería una ganancia ulterior o también un valor agregado), consiste en el hecho de que tal método favorece o acompaña el cambio o la transformación vivida en la misma comunidad, que además de valorizar hace emerger su calidad de ser comunidad de sujetos, 
que acompaña también a los mismos sujetos para estar siempre en formación. El discernimiento comunitario tiene como fin, en esta óptica, pensar acciones capaces de estar en el mundo con un buen sentido de responsabilidad. La acción que la misma planificación pastoral piensa y realiza debe estar orientada a dar forma a la comunidad. Podríamos decir que la comunidad cristiana se constituye en comunidad en la medida en que hace discernimiento, así se forma, lee su historia como historia de vida y de salvación y se mantiene en forma. La formación es el espacio vital con el que la comunidad asegura a su Señor, el Señor Resucitado. Discernir para planificar espacios formativos y condiciones correctas de aprendizaje al interno de la comunidad (condiciones-organizaciones, procesos, contenidos) es, entonces, para la Iglesia una cuestión vital: equivale a crear las condiciones para que el Señor la genere y re-genere continuamente. Podemos decir que la comunidad eclesial nace de un discernimiento formativo, si por formación entendemos precisamente la acción que el Señor Resucitado, a través de su Espíritu, pone en acto para conferirle continuamente su identidad (Biemmi, 2005).

Asumir la orientación narrativo-autobiográfica al interno del método ver-juzgar-actuar, a nivel del "ver" quiere decir, ahora más prácticamente, restituir la palabra a los sujetos. Para una planificación pastoral que desee realmente ser un instrumento de cambio no puede prescindir de involucrar a todos los sujetos interesados, llamados a aportar, no sólo la propia contribución en términos de ideas y propuestas, sino también en términos de vida, relatos de la propia historia y de la realidad. El método autobiográfico que hace referencia a la capacidad de cada quien, de relatar la propia historia de vida, consiente, en cuanto dispositivo heurístico, acoger la continuidad, la intencionalidad y lo significativo del propio recorrido de vida.

El sujeto, como yo fundamental en la construcción y planificación del propio itinerario de liberación, construye narración, cogiendo modos de representación de la realidad que tengan un significado socialmente compartido y nuevas representaciones de sí mismos. De esta manera los sujetos son así protagonistas profundos de cambios en torno a sí.

$\mathrm{Al}$ momento del "actuar", propio del método ver-actuar-juzgar, el sujeto se ve a sí mismo, realizando una representación de sí, y no es sólo observador. En este sentido el sujeto puede ser también, y, sobre todo, un sujeto eclesial.

El primer momento del método, el "ver", coincide con el recordar y recordarse para reportarse a las luces y conocer, actitudes no solo presentes en el primer momento de la planificación pastoral, sino ver en el acercamiento a la realidad, pero como motivación profunda que acompaña todo el camino de planificación. Mi realidad no es sólo mía, es el reflejo 
de un sujeto comunitario que se interroga sobre su propio futuro. No simple representatividad, uno a nombre de todos; es más bien el resultado, no como suma, sino como realidad nueva y diversa, de situaciones y contextos, que, así como han producido una realidad dolorosa en lo singular, como causa de sufrimiento en toda la comunidad, crea situaciones de liberación y superación personal y comunitaria por el principio de solidaridad y fraternidad. La superación catártica es una condición de posibilidad que es necesario realizarla y no descuidar en el momento del ver, como conocimiento de la realidad. El relato, la narración genera aquel desapegarse necesario en la aceptación y en la elaboración de los dolores vividos, al que sigue, inevitablemente el efecto curativo.

El sujeto, singular o comunitario, mientras narra y es escuchado en su observar, se hace autor, inicia a indicar recorridos de liberación que se realizarán y se historificarán, harán historia mediante la planificación pastoral. El sujeto es tal, ya sea porque está involucrado en el "ver", relatando las causas que le han llevado a situaciones de pobreza y esclavitud, ya sea por posibles autores o él mismo en historias nuevas.

El proceso de distanciamiento, como condición de posibilidad del conocer, es facilitado por los "heterónomos", del cual el narrador o relator se sirve para representar sentimientos o tratos de la personalidad, de otro modo no reconocibles o inaceptables. El contar la historia de vida permite encontrarse o conocer las múltiples identidades que habitan en cada uno de nosotros. No basta relatarse o relatar, es necesario que sean develados o reconocidos los procesos de narración, de modo que las personas aprenden alguna cosa más de sí mismas, de los otros o del mundo por construirse de nueva manera.

Es el pensamiento narrativo, en el cual se coloca el relato autobiográfico, el que favorece la construcción de modelos interpretativos de la realidad, que favorecen el aspecto semántico y transforman la experiencia en una historia coherente. El sujeto autobiográfico reconstruye la propia existencia, en modo arbitrario o subjetivo entorno a eventos de mercado (eventsmarkers) que lo han señalado y modificado, poniendo luz no en la realidad sino en una propia y particular realidad y, de este modo, los hechos se transforman en artefactos, a través de esta re-visitación creativa de la propia vida.

Hemos pasado por la experiencia del conocimiento en varios aspectos de su ser, según categorías hermenéuticas, y nos hemos dado cuenta de cuánta vivacidad y vida hay en la aproximación a la realidad, con sus reglas y sus tiempos.

El mismo "ver" junto a la "escucha" como actividad de Dios hacia su pueblo, visto en el libro del Éxodo, nos ha manifestado que también la vida de Dios en obediencia a sí mismo, vive un encuentro con la realidad 
humana, para transformarla y cambiarla; es la misma vida de Dios que se cruza con la realidad del hombre para hacer de ella una realidad nueva. Pero la vida, como nos recuerda Gabriel García Márquez (2002: 2): "no es la que uno vivió, sino la que uno recuerda y cómo la recuerda para contarla", por eso el nuevo paradigma que se quiere asumir en la planificación pastoral, y precisamente ampliando el método ver-juzgar-actuar, es el paradigma biográfico-narrativo, que se asume como un nuevo "ver" la vida, no sólo desde el individuo, sino desde toda una comunidad, como capacidad de entrar profundamente en la realidad, para acogerla y acompañarla a lo largo de todo el proceso de liberación y esperanza.

\section{Conclusión}

La presente investigación no pretende debilitar el método ver-juzgaractuar, sino más bien fortalecerlo y, sobre todo, fortalecerlo en el momento del "ver" con respecto a los instrumentos operativos capaces de frenar los riesgos decripto-reductivistas o programaciones desarrolladas en otros lugares, en otros contextos o mesas de café. Para que sea, como de hecho viene pensado, un instrumento realmente de análisis de una realidad profunda que muchas veces no alcanzamos a ver con el método y las técnicas del pasado, es necesario reflexionar sobre cómo las historias de vida puedan dar una aportación instrumental eficaz en orden a la planificación pastoral.

En el ámbito de las ciencias humanas, la importancia de las historias y de los relatos de vida es una realidad bien aclarada, tanto que ya no es necesario pedir muchas demostraciones. El éxito de las instancias narrativas es debido a la cercanía tan evidente hacia al hombre, ya que ésta toca la raíz de una de las dimensiones fundamentales de la existencia humana: el relato. En el ámbito teológico, sobre todo la catequesis y la pastoral, son las disciplinas que han hecho propia, más que otras, esta instancia antropológica. Sin embargo, la reflexión teológica está distante del haber tomado conciencia de las implicaciones que resultan del redescubrimiento del relato.

El profesor Elmar Salmann (2012: 3) escribe cómo "no hay biografía sin una pizca de teología y antropología". Escribir, narrar, volver a trazar el hilo de su propia vida o de la existencia de los demás, significa "crear un mito, un relato, para ver y capturar un símbolo, un sentido, una metáfora abierta" (Salmann, 2012: 3). Cada vida, de hecho, es menos de cuanto es y aparece, y cada vida es aún más. Hay una biografía ontológica y teológica que se potencia en los numerosos encuentros que se realizan en el curso de la vida. De lo contrario no hay teología sin biografía. Así nace la fe y, 
como consecuencia, la capacidad de pensarla en un lento proceso de iniciación, de aprendizaje, de conversión.

Para alcanzar una reciprocidad entre narración y conocimiento de la realidad socio-eclesial, se necesitará un largo camino de iluminación recíproca, caracterizado por momentos de revisión, de conversión, de lectura y de cambio (Salmann, 2012). Es por eso que al centro de un pensamiento, de una misión y de lo vivido, hay con frecuencia un cambio, un éxodo (Dios que habla a su pueblo), un encuentro (entre Jesús y sus discípulos), un impacto y una conversión (san Pablo), una iluminación (san Agustín).

El paradigma narrativo pide a la planificación pastoral que se equipe, se refuerce, con una metodología de tipo fenomenológico, que asuma como objeto de reflexión lo vivido eclesial, y que revea sus finalidades según una óptica de un servicio decididamente formativo.

Según algunos autores (Alheit \& Bergamini, 1996), la narración con las historias de vida son y hacen formación porque se orientan necesariamente hacia la "autoconciencia" y la "transformación". De esta manera se activa el ejercicio de la reflexividad, que se realiza en el discutir con otros y que opera el cambio de los puntos de vista, la toma de distancia afectiva, el desapego del propio pasado, del presente y del futuro.

La teología debe preguntar, suscitar, evocar, dejar hablar, narrar y escribir la vida de las personas de modo que pueda nacer una experiencia nueva también en el ámbito teológico y eclesial. De manera más evidente, la línea de demarcación hacia donde nos empuja el paradigma narrativo está en el proponer a la teología: o bien continuar con los conceptos metafísicos con metodologías lógico-argumentativas, o trabajar con experiencias en clave fenomenológica. Frente a esta encrucijada, nosotros con Scaramuzzi (2011) preferimos una interpretación de tipo fenomenológico, anti-positivista, más adecuada respecto al fin que nos proponemos, y porque reconoce al lenguaje una función fundamental en la "construcción" de la realidad. El lenguaje no es un médium neutral a través del cual la experiencia es llevada a palabra comunicada, sino es la forma misma a través de la cual la experiencia es configurada; es decir, interpretada y modificada del sujeto y del por el ambiente cultural de su contexto de pertenencia. La narración es un acto performativo, por medio del cual el sujeto configura y atribuye sentido a sus vivencias propias. En esta luz se entenderá, entonces, la perspectiva de significado que asume el paradigma narrativo en el orden de la praxis y de la planificación pastoral: es un trabajar con historias y no con conceptos. Si, de hecho, la orientación es trabajar con experiencias y con lo vivido, se necesita llevar a primer plano las Historias de vida de las comunidades, que podríamos llamar las historias de Iglesia, con todos sus relatos de ordinaria vida eclesial, historias de un pueblo con todos sus logros y conflictos, y tomarlas como objeto proprio y caracterizador 
de una teología en forma narrativa.

El "ver", como parte de la trilogía ver-juzgar-actuar, es la experiencia del conocer por lo que se ha visto y lo que se ha escuchado; lo primero hace referencia a la metodología cuantitativa, y lo segundo a una cualitativa; lo primero se manifiesta a través de las estadísticas, y lo segundo se revela a través de las historias narrativas y de la vida.

Antes de un proyecto por realizar se necesita escucha por pensar; sin la escucha simpática e interesada, el mismo "ver" se puede convertir en un acto de violencia de la realidad. Por eso nos parece apropiado, hablando del método de planificación pastoral ver-juzgar-actuar, todavía valido siempre y cuando prevea un ver "cálido", que sepa entrar en relación con la realidad sin vulnerarla; además, el mismo escuchar no tiene que terminarse en la primera fase de ejecución del proceso metodológico, más bien tendría que acompañarlo en cada etapa de su actuación, si es verdad que no hay cambio si no hay aceptación del otro, y no hay aceptación del otro si no hay escucha.

\section{REFERENCIAS}

-Alheit, P. \& Bergamini, S. (1996). Storie di vita. Metodología di ricvercaper le scienze sociali. Milano: Guerini.

-Arrieta, R. C. (2009). Habermas y la teoría de la acción comunicativa. Recuperado desde http://iusconstifil.blogspot.com/2009/04/habermas-y-la-teoria-de-la-accion.html.

-Biemmi, E. (2005). Pietra che cammina. Diventare comunità oggi. Scienze umane e teologia pastorale in dialogo per una ricerca-azione nelle Chiese di Puglia. Monopoli (BA): Vivere In.

-Bodei, R. (2001). La filosofia del siglo XX. Madrid: Alianza.

-Brighenti, A. (1994). Metodología para un proceso de planeación participativa. Bogotá: Paulus.

-Brighenti, A. (2003). Recostruyendo la Esperanza. Cómo planear la acción de la iglesia en tiempos de cambio. México, D.F.: Palabra ediciones.

-Castillo, R. B. (2004). Ponderación teológica del método Ver-Juzgar-Actuar. ITER (34), 19-52.

-Demetrio, D. (1996). Raccontarsi. L'autobiografia come cura di sé. Milano: Cortina Raffaello.

-Gadamer, H. G. (2000). Verità e metodo. Milano: Bompiani.

-García Marquez, G. (2002). Vivir para contarla. Barcelona: Mondadori.

-Heidegger, M. (1927). Sein und Zeit. Germania: Halle.

-Heidegger, M. (1968). Sentieri interrotti. Firenze: La Nuova Italia.

-Klein, S. (1999). Methodische Zugängezursozialen Wirklichkeit. In H. Haslinger (Hg), HandbuchPraktischeTheologie (Band 1. pp. 248-259). Grundlegungen: Mainz. 
-Lanza, S. (2010). La teologia pastorale secondo la scuola lateranense. I Laterani. Roma: LUP.

-Leyte, A. (2006). Heidegger. Madrid: Alianza.

-Mette, N. (1978). Theorie der Praxis. Germany: Düsseldorf.

-Muzzetto, L. (2011). L'intersoggettivitá e la sfera "emozionale-affettiva". Societá Mutamento Politico, 2, 65-100. Revista online www.fupress.net.

-Salmann, E. (2012). Biografia e teologia: due parole dell'infinito. Rivista di Scienze Religiose, XXVI(1), 3-5.

-Sartre, J. P. (1960). Critique de la raison dialectique. Questions de méthode. Paris: Gallimard.

-Scaramuzzi, D. (2011). Fare Chiesa. Saggi di aggiornamento ecclesiologico. San Giovanni Rotondo (Fg): Sao Ko Kelle Terre.

-Schütz, A. (1974). La fenomenologia del mondo sociale. Bologna: Il Mulino.

-Sini, C. (1986). I filosofi e le opere. L'etá contemporanea (Vol. III). Milano: Principato.

-Vela, J. A. (1986). El proceso de planificación participativa. Bogotá: Casa de la Juventud.

Sumario: Introducción; 1. La planeación participativa: origen y momentos; 2. Método participativo y magisterio eclesiástico; 3. Límites del método participativo; 4. Consideraciones útiles para superar los límites; 5. Las historias de vida: una propuesta para enriquecer el método participativo; Conclusión; Referencias. 